\title{
Shall We Use Hydroxychloroquine in Hemodialysis Patients?
}

\author{
Ahmet Murt Mevlut Tamer Dincer Cebrail Karaca \\ Department of Internal Medicine/Division of Nephrology, Cerrahpasa Medical Faculty, Istanbul \\ University-Cerrahpasa, Istanbul, Turkey
}

Dear Editor,

Shall we use hydroxychloroquine (HQ) in hemodialysis patients?

We welcome the comments by Wiwanitkit [1] made on our case report. In another COVID-19 study with small sample size of 21 hemodialysis patients, no HQrelated arrhythmia was reported, but ECG monitoring revealed prolongation of corrected QT intervals [2]. Since hemodialysis patients have high cardiac risks, arrhythmogenic drugs like HQ should be used with caution.

A clear-cut boundary to attribute the arrhythmia to a specific condition or a drug may not be always possible for hemodialysis patients. Hypoxemia, thromboemboli, or cardiac complications may further blur the arrhythmia etiology in COVID-19 patients. However, we could not put the blame on COVID-19 in the presented cases as patients' clinical conditions were improving when sudden cardiac death occurred. Acute coronary syndrome was also ruled out in both cases. Electrolyte shifts may be blamed to some extent and HQ had most probably eased the arrhythmia generation.

We might not establish a direct causal relationship with just 2 cases, and this was not the intention. Considering cardiac risk factors for hemodialysis patients, in light of the direct mortality due to COVID-19, we should continue HCQ as used in sequenced multidrug therapy, which has been associated with large reductions in hospitalization and death when initiated in the early ambulatory phase [3-5]. Strategies to mitigate HCQ-related arrhythmia risk have been discussed in the original case report [6].

\section{Conflict of Interest Statement}

The authors declare no conflicts of interest.

\section{Funding Sources}

The authors did not receive any funding.

\section{Author Contributions}

A.M. did the literature search, wrote the first draft, and coordinated the discussion with other authors. M.T.D. and C.K. helped in literature search and discussed the content of the text. All the authors commented and agreed on the final version of the manuscript.
Correspondence to:

Ahmet Murt, ahmet.murt@ istanbul.edu.tr 


\section{References}

1 Wiwanitkit V. Sudden cardiac death, haemodialysis, hydroxychloroquine, and COVID19. Blood Purif. DOI: 10.1159/000515010.

2 Giaime P, Guenoun M, Pedinielli N, Narbonne $\mathrm{H}$, Bergounioux JP, Solas C, et al. Hydroxychloroquine and azithromycin tolerance in haemodialysis patients during $\mathrm{CO}$ VID-19 infection. Nephrol Dial Transplant. 2020 Aug;35(Issue 8):1346-53.

3 McCullough PA, Alexander PE, Armstrong $\mathrm{R}$, Arvinte C, Bain AF, Bartlett RP, et al. Multifaceted highly targeted sequential multidrug treatment of early ambulatory high-risk SARS-CoV-2 infection (COVID-19). Rev Cardiovasc Med. 2020 Dec 30;21(4):517-30.
4 Procter BC, Ross C, Pickard V, Smith E, Hanson $\mathrm{C}$, McCullough PA. Early ambulatory multidrug therapy reduces hospitalization and death in high-risk patients with SARSCoV-2 (COVID-19). Authorea. 2021 Jan 07.

5 Derwand R, Scholz M, Zelenko V. COVID-19 outpatients: early risk-stratified treatment with zinc plus low-dose hydroxychloroquine and azithromycin: a retrospective case series study. Int J Antimicrob Agents. 2020 Dec; 56(6): 106214.

6 Murt A, Dincer MT, Karaca C. Sudden cardiac death in haemodialysis patients under hydroxychloroquine treatment for $\mathrm{CO}$ VID-19: a report of two cases. Blood Purif. 2020 Oct 8:1-3. 